\title{
Optimization of Sensor, Bus, and Fusion Schedules of a Time-Triggered Sensor Fusion System
}

\author{
Moritz Mauthner, Richard Altendorfer, Wilfried Elmenreich and Alexander Kirchner
}

\begin{abstract}
In this paper we optimize a time-triggered, Kalman filter based, multi-sensor fusion system, used as an environmental perception platform for advanced driver assistance systems while satisfying constraints that are typical of a safety-related application.

We argue that the overall system including effects from the sensor, bus, and fusion schedules as well as the treatment of measurements must be considered in order to optimize the fusion accuracy. Due to differences in measurement preprocessing data from sensors may not arrive in chronological order which requires special treatment for this out-of-sequence measurements (OOSM). As a result of this paper we identify regions in the scheduling parameter space that minimize the error covariance of the estimated states.
\end{abstract}

\section{INTRODUCTION}

Multi-sensor data fusion systems are the enabling technology for advanced driver assistance systems. New features like adaptive cruise control with active steering, lane departure alert, parking pilot, and automatic emergency brake will be standard in the next car generation as electronic stabilization systems and anti lock braking systems are at present.

This paper deals with the optimization of a time-triggered, Kalman filter based, sensor fusion system for driver assistance systems consisting of two sensors, a bus system and a fusion/prediction core. It tracks objects and is supposed to deliver a real time image (model) of the environment at fixed intervals with given accuracy to a safety related application (see figure 1).

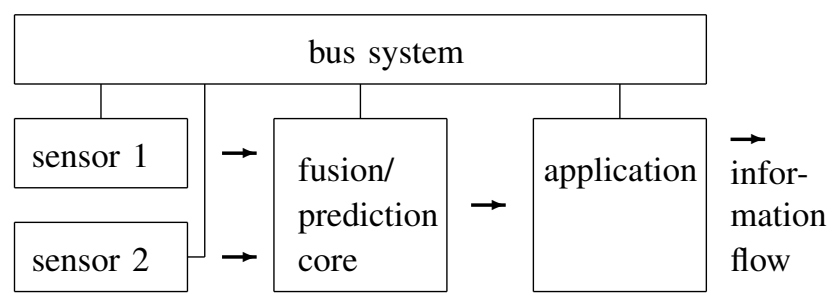

Fig. 1. Information flow in sensor fusion

The rest of the paper is structured as follows: In section II the system set-up is described. In section III OOSM

M. Mauthner is with Volkswagen AG, Wolfsburg, Germany moritz.mauthner@volkswagen. de

R. Altendorfer is with Audi Electronics Ventures GmbH, Ingolstadt, Germany richard.altendorfercaudi.de

W. Elmenreich is with the Department of Computer Engineering, Vienna University of Technology, Vienna Austria wilevmars.ac.at

A. Kirchner is with Volkswagen AG, Wolfsburg, Germany alexander.kirchner@volkswagen. de treatment strategies are discussed. Section IV presents the optimization approach. We analyse the optimal parameter regions in section V. In section VI a conclusion is drawn.

\section{SYSTEM DESCRIPTION}

We consider a system with two identical sensors that transmit their measurements via a time-triggered bus system to a fusion/prediction core. The fusion/prediction core fuses the incoming measurements and then predicts the fused object states to real time as demanded by an application (figure 1). We use the following definitions:

- the cycle time $t_{C}$ of a process is the time interval that this process requires for completion of its service while the start of two consecutive processes is $t_{C}$ apart

- the execution time $t_{T}$ of a process is the time interval in which this process completes its service where the start of two consecutive processes can be more than $t_{T}$ apart

- the phase $t_{P}$ of a process is defined as the time interval between the start of this process relative to the start of the first cycle of sensor 1

The two sensors have cycle times $t_{C}^{\text {sens } 1}$ and $t_{C}^{\text {sens } 2}$, that are identical to their preprocessing times and therefore adjustable by the choice of the speed of the sensor's processor. They deliver their measurement states $\vec{z}_{t_{k}}^{\text {sens } 1}$ and $\vec{z}_{t_{k}}^{\text {sens } 2}$ with constant covariance matrices $R_{t_{k}}^{\text {sens } 1}$ and $R_{t_{k}}^{\text {sens } 2}$ over a time-triggered bus system using time division multiple access (TDMA) with fixed cycle time $t_{C}^{t t a}$ (the bus is not exclusively used by the sensor fusion system, hence the cycle time is assumed to be determined by external constraints) to a fusion/prediction core. There the information is processed in order to establish a robust image of the environment.

In figure 2 the cycle times, slots for execution times and phases of sensor preprocessing $\left(t_{C}^{\text {sens } 1}, t_{C}^{\text {sens } 2}\right.$ and $\left.t_{P}^{\text {sens }}\right)$, measurement transmission $\left(t_{C}^{t t a}, t_{P}^{t t a 1}\right.$ and $\left.t_{P}^{t t a 2}\right)$ and generation of the application update $\left(t_{C}^{p r e}, t_{T}^{p r e}\right.$ and $\left.t_{P}^{p r e}\right)$ that are processed within the fusion system are visualized.

To process the incoming measurements at the fusion/prediction core, we use a Kalman filter approach as described in [1]. As the Kalman filter is a widely used and well known algorithm for tracking moving targets, we will only give a short overview of the notation used throughout this paper. The estimated state vector of the tracked object $\hat{\vec{x}}\left(t_{k-1} \mid t_{k-1}\right)$ is updated with measurements at discrete points in time $t_{k}$. The state prediction from $t_{k-1}$ to $t_{k}$ is given by $\hat{\vec{x}}\left(t_{k} \mid t_{k-1}\right)=F\left(t_{k}-t_{k-1}\right) \cdot \hat{\vec{x}}\left(t_{k-1} \mid t_{k-1}\right)$. The predicted state covariance matrix is computed as 


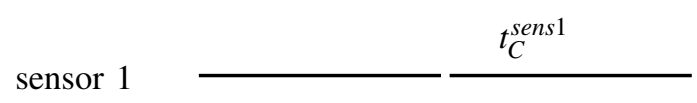

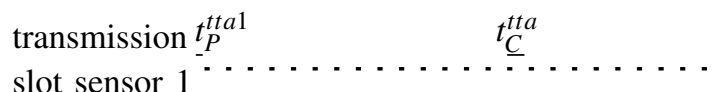

sensor $2 \quad \underline{t_{P}^{\text {sens }}} \stackrel{-}{-}-{ }_{C}^{\text {sens } 2}$

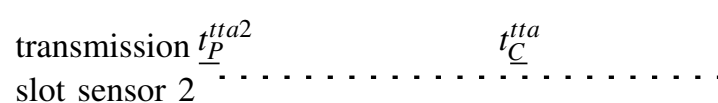

slot for

fusion task ---------

$\begin{array}{llll}\text { prediction } & t_{P}^{\text {pre }} & t_{T}^{\text {pre }} & \frac{t_{C}^{\text {pre }}}{-}-\underline{-}_{-} \\ \text {cycle } & - & - & -\end{array}$

real time

Fig. 2. System schedule

$P\left(t_{k} \mid t_{k-1}\right)=F\left(t_{k}-t_{k-1}\right) \cdot P\left(t_{k-1} \mid t_{k-1}\right) \cdot\left(F\left(t_{k}-t_{k-1}\right)\right)^{T}+$ $Q_{t_{k-1}}$. The measurement update of the predicted system state is $\hat{\vec{x}}\left(t_{k} \mid t_{k}\right)=\hat{\vec{x}}\left(t_{k} \mid t_{k-1}\right)+K_{t_{k}} \cdot \vec{v}_{t_{k}}$ with $K_{t_{k}}=P\left(t_{k} \mid t_{k-1}\right) \cdot H_{t_{k}}^{T}$. $\left(H_{t_{k}} P\left(t_{k} \mid t_{k-1}\right) H_{t_{k}}^{T}+R_{t_{k}}\right)^{-1}, \vec{v}_{t_{k}}=\vec{z}_{t_{k}}-\hat{\vec{z}}_{t_{k}}$ and $\hat{\vec{z}}_{t_{k}}=H_{t_{k}} \cdot \hat{\vec{x}}\left(t_{k}\right)$. The update of the covariance matrix is given by $P\left(t_{k} \mid t_{k}\right)=$ $\left(I-K_{t_{k}} \cdot H_{t_{k}}\right) \cdot P\left(t_{k} \mid t_{k-1}\right) \cdot\left(I-K_{t_{k}} \cdot H_{t_{k}}\right)^{T}+K_{t_{k}} \cdot R_{t_{k}} \cdot K_{t_{k}}^{T}$. The fusion/prediction core processes a measurement within $t_{T}^{\text {fus }}$.

\section{Out-OF-SEQuence MeAsurement Treatment}

In multi-sensor tracking systems, measurements from the same object can arrive out of sequence [2], [3], that means not in chronological order. Often, OOSM behavior is caused by an indeterministic transmission system, where the transmission time of a message may vary so much that a message from a later measurement may overtake a newer measurement. Such behavior is caused by transmission protocols with many retries such as many Internet protocols (e. g., TCP/IP) or in networks with dynamic routing (Internet, wireless sensor networks).

However, even if communication protocols with deterministic behavior, such as time-triggered approaches like flexray [4], TTCAN [5], TTP [6], or TTP/A [7] are used, the OOSM problem may arise.

Figure 3 depicts a situation with an OOSM problem that is independent from communication system issues. Due to different preprocessing times the indicated measurement from sensor 2 is received earlier at the fusion core than the indicated measurement from sensor 1 although the measurement from sensor 2 is newer.

As the Kalman filter in the form of [1] can not handle OOSMs, architectural and algorithmic solutions have been developed.

Kaempchen et al. [8] use a measurement buffer between bus and fusion core. No measurement is delivered to the fusion/prediction core, before all measurements that have been sampled earlier have arrived at the buffer. Within the buffer

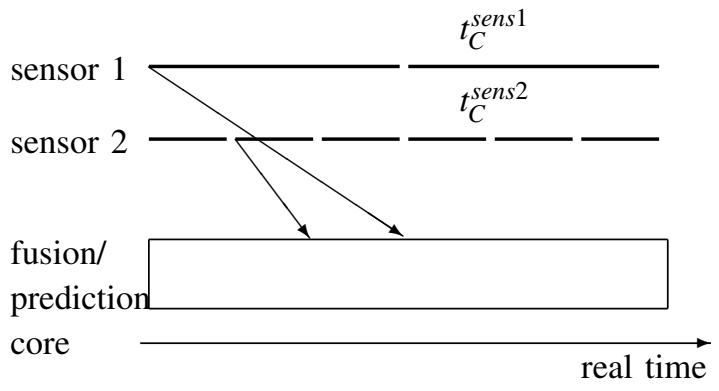

Fig. 3. Origin of out-of-sequence measurements

the measurements are sorted chronologically. Kaempchen et al. further discuss the maximum latency (here defined as the time difference between the instant of the composition of the image representing the surrounding environment provided by sensor fusion and the bygone instant where this image was true) that arises between measurement recording and measurement fusion. It is distinguished between situations where only knowledge of the maximum measurement cycle times and situations where full knowledge of the measurement cycle times is available.

Another way to solve the OOSM problem are special algorithms which will be called "advanced algorithms" throughout this paper. These algorithms deal with one-lag and multi-lag delays, filtering and tracking, linear and nonlinear systems as well as single-model and multi-model approaches [9], [10], [11], [12], [13], [14], [15], [16], [17], [18].

For sake of discussion we will only consider the buffering approach as presented in [8] referred to as $B U F F$ and the OOSM treatment algorithm A $l 1$ of [10] referred to as $A D V A$.

\section{OPTIMIZATION APPROACH}

As the application is safety related, it expects to periodically receive (cycle time $t_{C}^{\text {pre }}$ ) real time object states with bounded accuracy. Due to the fact that the fusion output states always lag behind real time irrespective of the specific OOSM algorithm used, the image of the environment has to be predicted from state time $t_{S T}$ to real time $t_{R T}$. The state time is the current time of the Kalman filter state and coincides with the measurement time of the latest fused sensor measurement, whereas real time is the actual physical time. The real time covariance matrix of the state can be determined by predicting the state time covariance matrix from state time $t_{S T}$ to real time $t_{R T}$ by $P\left(t_{R T} \mid t_{S T}\right)=$ $F\left(t_{R T}-t_{S T}\right) \cdot P\left(t_{S T} \mid t_{S T}\right) \cdot\left(F\left(t_{R T}-t_{S T}\right)\right)^{T}+Q_{S T}$ (see figure 4).

As the dependence of $F\left(t_{R T}-t_{S T}\right)$ and $Q_{S T}$ on the prediction interval is well known for a given system, it is thus possible to consider the effect of the system parameters and the strategy for the treatment of OOSM (see also section III) on the system latency $t_{R T}-t_{S T}$, state time and real time covariance matrices of the state $P\left(t_{S T} \mid t_{S T}\right)$ and $P\left(t_{R T} \mid t_{S T}\right)$.

The task is to analyse the effect of different OOSM treatments and parameter sets on the accuracy (defined as 


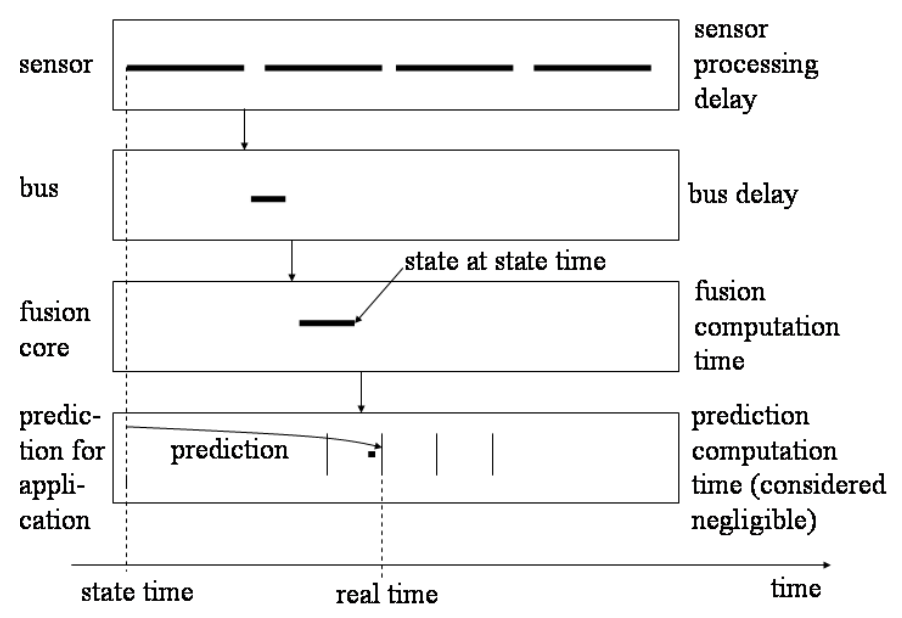

Fig. 4. Prediction from state time to real time

the determinant of the real time covariance matrix, after the Kalman filter has converged).

The optimization will be performed by simulating the entire system including bus and sensor schedules at given points in parameter space. We use a simple dynamic system and measurement model in the fusion/prediction core (see appendix) and employ a Kalman filter (by linearization around a setpoint) as opposed to an extended Kalman filter as our estimation algorithm. As the computation of the covariance matrices of a Kalman filter is completely independent of the state and measurement vectors we need not input specific measurements and are thus independent of the actual driving situation. After convergence of the Kalman filter ${ }^{1}$ the maximum of $P\left(t_{R T} \mid t_{S T}\right)$ is recorded. This constitutes one iteration of the optimization (minimization of $\left.\operatorname{det}\left(P\left(t_{R T} \mid t_{S T}\right)\right)\right)$ over the regions of the parameter space.

We will discuss both the area of the covariance ellipse at state time $\operatorname{det}\left(P\left(t_{S T} \mid t_{S T}\right)\right)$ as well as at real time $\operatorname{det}\left(P\left(t_{R T} \mid t_{S T}\right)\right)$. With the system latency $\Delta t=t_{R T}-t_{S T}$, $\operatorname{det}\left(P\left(t_{R T} \mid t_{S T}\right)\right)$ is given by

$$
\begin{array}{r}
\operatorname{det}\left(P\left(t_{R T} \mid t_{S T}\right)\right)= \\
\operatorname{det}\left(F(\Delta t) \cdot P\left(t_{S T} \mid t_{S T}\right) \cdot F(\Delta t)^{\top}+Q_{S T}\right)= \\
\operatorname{det}\left(P\left(t_{S T} \mid t_{S T}\right)\right)+\Delta t \cdot P\left(t_{S T} \mid t_{S T}\right)(1,1) \cdot q+O\left(\Delta t^{2}\right)
\end{array}
$$

This allows us to separate the influence of the sensor schedules and the OOSM algorithms that affect the covariance at state time $P\left(t_{S T} \mid t_{S T}\right)$ and the influence of the bus, fusion and prediction schedules. From eq. 1 we see that given $\operatorname{det}\left(P\left(t_{S T} \mid t_{S T}\right)\right)$ the leading order increase of the covariance area at real time is linear in $\Delta t$. As will be demonstrated in section $\mathrm{V}$, the variations in $P\left(t_{S T} \mid t_{S T}\right)$ are negligible and it

\footnotetext{
${ }^{1}$ Convergence of the Kalman filter here means that the covariance matrices have assumed a regular, periodic pattern. A constant steady state covariance matrix cannot be expected since the Kalman filter receives measurements from different sensors at different times.
}

then suffices to minimize $\Delta t$ in order to maximize the fusion accuracy at real time.

\section{Optimal Parameter Regions}

As $t_{C}^{t t a}$ and $t_{C}^{p r e}$ are fixed (see sections II and IV), $t_{C}^{\text {sens } 1 \text {, }}$ $t_{C}^{\text {sens } 2}, t_{T}^{f u s}$ and $t_{T}^{\text {pre }}$ are the only system parameters whose modification changes the hardware of the system and affects such the overall system costs. In contrast, $t_{P}^{\text {sens }}, t_{P}^{t t a 1}, t_{P}^{t t a 2}$ and $t_{P}^{p r e}$ can be modified without affecting the costs of the system by simply changing software parameters. Therefore, for every configuration of $t_{C}^{\text {sens } 1}, t_{C}^{\text {sens } 2}, t_{C}^{t t a}, t_{T}^{f u s}$ and $t_{T}^{p r e}$, we choose the phases that minimize the maximum of $\operatorname{det}\left(P\left(t_{R T} \mid t_{S T}\right)\right)$ which has been computed numerically over a simulation time of 20 seconds performed with a simple dynamic system and measurement model (see appendix).

Furthermore we consider the algorithmic overhead of the advanced algorithms in comparison to the simple buffering approach by adding a penalty term to $t_{T}^{f u s} A D V A=t_{T}^{f u s}+$ $t_{T}^{\text {Overhead }} . t_{T}^{\text {Overhead }}$ was empirically determined as $t_{T}^{\text {Overhead }} \approx$ $t_{T}^{f u s} \cdot t_{T}^{\text {pre }}$ is approximated by $t_{T}^{\text {pre }} \approx \frac{t_{T}^{f u s}}{3}$. Note that all temporal parameters are varied in $1 \mathrm{~ms}$ steps.

In figures 5 and 6 we visualize the statetime determinant of the error covariance matrix $\min _{t_{P} t a 1, t_{P}^{t a 2}}\left(\max _{t}\left(\operatorname{det}\left(P\left(t_{S T} \mid t_{S T}\right)\right)\right)\right) \quad$ and the realtime determinant of the error covariance matrix $\min _{t_{P} t a 1, t t_{P} a 2}\left(\max _{t}\left(\operatorname{det}\left(P\left(t_{R T} \mid t_{S T}\right)\right)\right)\right)$ for $t_{C}^{\text {sens } 1}=50 \mathrm{~ms}$, $t_{C}^{\text {sens } 2}=40 \mathrm{~ms}, t_{C}^{\text {tta }}=2 \mathrm{~ms}, t_{T}^{\text {fus }}=1 \mathrm{~ms}$ and $t_{C}^{\text {pre }}=30 \mathrm{~ms}$ by variation of $t_{P}^{\text {sens }}$ and $t_{P}^{\text {pre }}$ for both advanced algorithms and buffering.

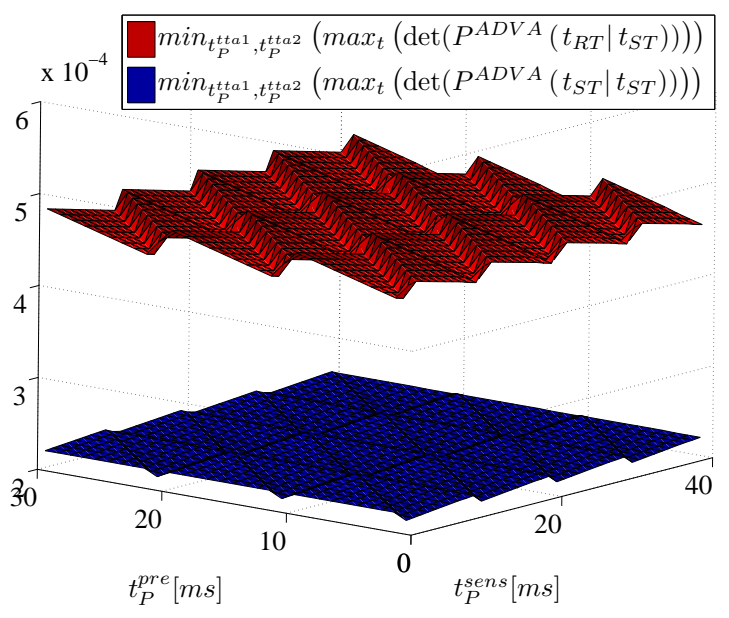

Fig. 5. State-time and real-time accuracy of the advanced algorithms approach for $t_{C}^{\text {sens } 1}=50 \mathrm{~ms}, t_{C}^{\text {sens } 2}=40 \mathrm{~ms}$

As $R_{t_{k}}^{\text {sensor } 1}$ and $R_{t_{k}}^{\text {sensor } 2}$ are constant in time (see appenix) and the variation of $\min _{t_{P} t a 1, t_{P} t a 2}\left(\max _{t}\left(\operatorname{det}\left(P\left(t_{R T} \mid t_{S T}\right)\right)\right)\right)$ is negligible (as can be seen in figures 5 and 6), the influence of the prediction from state time $t_{S T}$ to real time $t_{R T}$ dominates all other factors. Given that the variability of the state time covariance is seen to be small, equation 1 shows that the covariance at real time is linearly determined by the prediction 


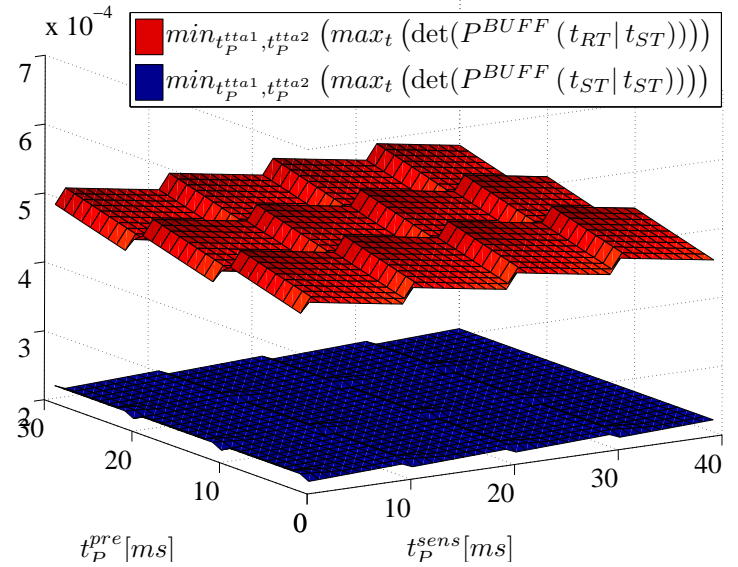

Fig. 6. State-time and real-time accuracy of the buffering approach for $t_{C}^{\text {sens } 1}=50 \mathrm{~ms}, t_{C}^{\text {sens } 2}=40 \mathrm{~ms}$

time interval that is visualized for the buffering approach in figure 7 , which shows the same structural behavior as the upper surface in figure 6 .

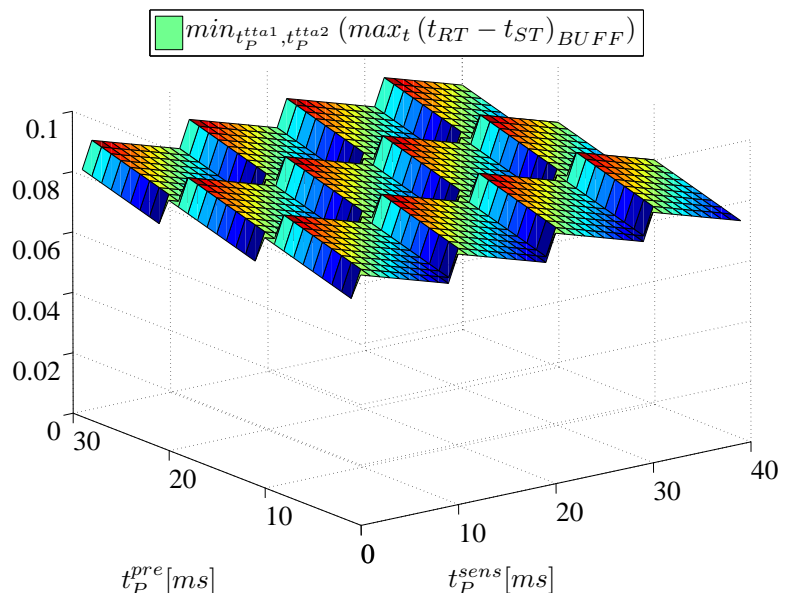

Fig. 7. $t_{R T}-t_{S T}$ profile for buffering approach for $t_{C}^{\text {sens } 1}=50 \mathrm{~ms}, t_{C}^{\text {sens } 2}=$ $40 \mathrm{~ms}$

In order to understand the periodic structural behavior, it is important to note that every parameter set $\left(t_{C}^{\text {sens } 1}, t_{C}^{\text {sens } 2}\right.$, $t_{P}^{\text {sens }}, t_{C}^{t t a}, t_{P}^{t t a 1}, t_{P}^{t t a 2}, t_{C}^{p r e}, t_{T}^{\text {pre }}$ and $\left.t_{P}^{p r e}\right)$ results in a series of prediction intervals from state time to real time $t_{R T}-t_{S T}$ that is periodically repeated over the simulation time with a periodicity determined by the least common multiple of all cycle times $t_{C}$. The shift between two cyclic processes is changing as well over simulation time and forms a shift series, where two consecutive shifts differ by $t_{C}^{1}-t_{C}^{2}$ or $\min \left(t_{C}^{1}, t_{C}^{2}\right)$. From every shift series one prediction interval series can be derived. If the phase is varied by $n \cdot\left(t_{C}^{1}-t_{C}^{2}\right)$ ( $n$ integer) it can happen, that the shift series has the same set of shifts and only starts at a different shift element. As only the maximum of $t_{R T}-t_{S T}$ is important to us it does not matter at which shift element our simulation starts. As $t_{C}^{\text {sens } 1}-t_{C}^{\text {sens } 2}=10 \mathrm{~ms}$ and $t_{C}^{\text {sens } 2}-t_{C}^{\text {pre }}=10 \mathrm{~ms}$ and we observe a $10 \mathrm{~ms}$ periodicity by variation of $t_{P}^{\text {sens }}$ as well as $t_{P}^{\text {pre }}$ this "10 ms shift invariance" is the case this parameter set.

The diagonal edges of the advanced algorithms approach result from the fact that a measurement is processed just before the prediction to real time is performed. Therefore the prediction interval from real time to state time is smaller which results in increased accuracy. The difference to the buffering approach is due to the fact that the measurement buffer influences the real time prediction interval profile in comparison to the advanced algorithms by delaying the measurements from the faster sensor, which partly compensates the change in $t_{P}^{\text {sens }}$.

In figures 8 and 9 we visualize the statetime determinant of the error covariance matrix $\min _{t_{P} t a 1, t t a 2}\left(\max _{t}\left(\operatorname{det}\left(P\left(t_{S T} \mid t_{S T}\right)\right)\right)\right) \quad$ and the realtime determinant of the error covariance matrix $\min _{t_{P}^{t t a 1}, t_{P}^{t t a 2}}\left(\max _{t}\left(\operatorname{det}\left(P\left(t_{R T} \mid t_{S T}\right)\right)\right)\right)$ for $t_{C}^{\text {sens } 1}=51 \mathrm{~ms}$, $t_{C}^{s e n s}=40 m s, t_{C}^{t t a}=2 m s, t_{T}^{f u s}=1 m s$ and $t_{C}^{p r e}=30 m s$ by

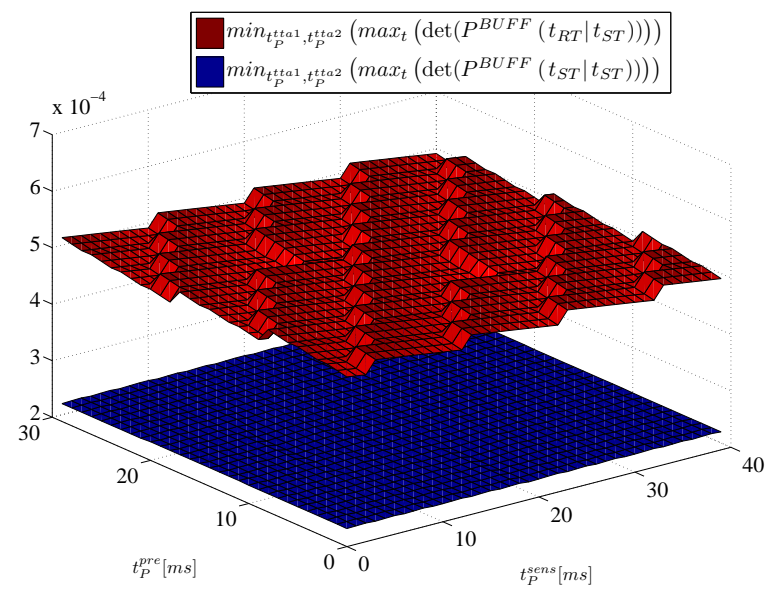

Fig. 8. State-time and real-time accuracy of the buffering approach for $t_{C}^{\text {sens } 1}=51 \mathrm{~ms}, t_{C}^{\text {sens } 2}=40 \mathrm{~ms}$

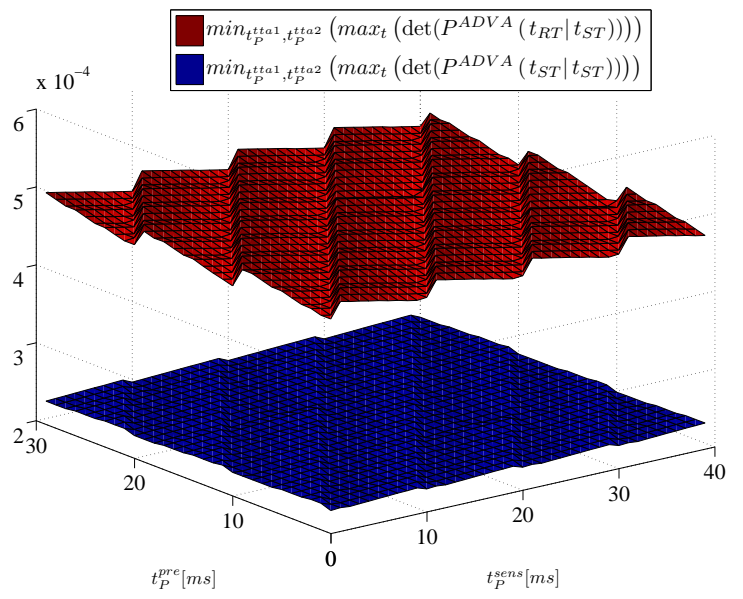

Fig. 9. State-time and real-time accuracy of the advanced algorithms approach for $t_{C}^{\text {sens } 1}=51 \mathrm{~ms}, t_{C}^{\text {sens } 2}=40 \mathrm{~ms}$ 
variation of $t_{P}^{\text {sens }}$ and $t_{P}^{p r e}$ for both buffering and advanced algorithms.

We see that the periodic structure disappears if $t_{C}^{\text {sens } 1}$ $t_{C}^{\text {sens } 2} \neq t_{C}^{\text {sens } 2}-t_{C}^{\text {pre }}$

As far as the influence of $t_{P}^{t t a 1}$ and $t_{P}^{t t a 2}$ is con-

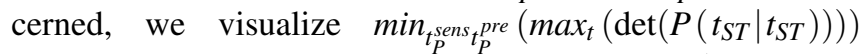
and $\min _{t_{P}^{\text {sens }} t_{P}^{\text {pre }}}\left(\max _{t}\left(\operatorname{det}\left(P\left(t_{R T} \mid t_{S T}\right)\right)\right)\right)$ for $t_{C}^{\text {sens } 1}=50 \mathrm{~ms}$, $t_{C}^{\text {sens } 2}=40 \mathrm{~ms}, t_{C}^{t t a}=2 \mathrm{~ms}, t_{T}^{\text {fus }}=1 \mathrm{~ms}$ and $t_{C}^{\text {pre }}=30 \mathrm{~ms}$ by variation of $t_{P}^{t t a 1}$ and $t_{P}^{t t a 2}$ for both buffering and advanced algorithms in figures 10 and 11 . Please note, that there exist two transmission schedule versions 1 and 2 . In version 1 sensor 1 sends in the first slot and sensor 2 sends in the second slot. In version 2 it is vice versa.

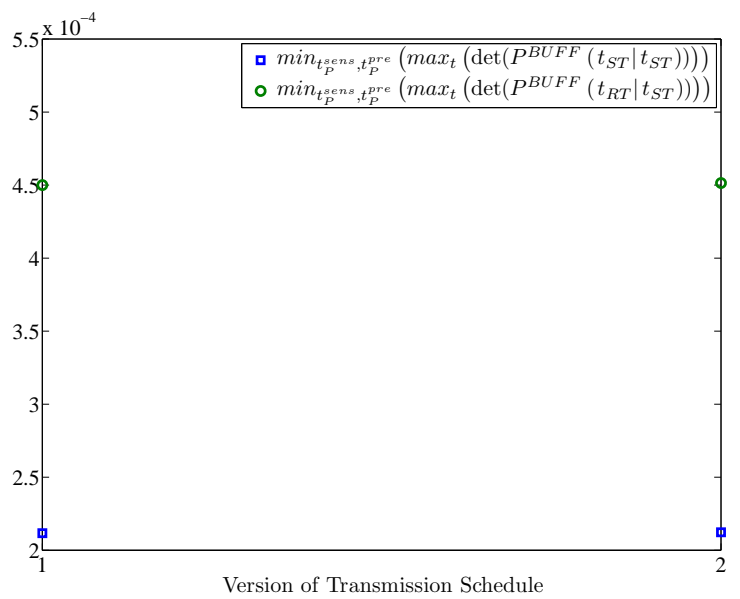

Fig. 10. State-time and real-time accuracy of the buffering approach for $t_{C}^{\text {sens } 1}=50 \mathrm{~ms}, t_{C}^{\text {sens } 2}=40 \mathrm{~ms}$

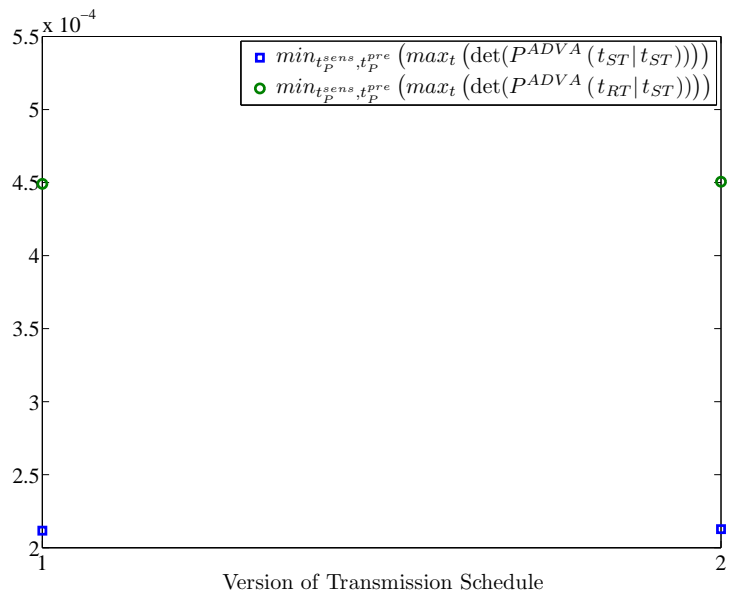

Fig. 11. State-time and real-time accuracy of the advanced algorithms approach for $t_{C}^{\text {sens } 1}=50 \mathrm{~ms}, t_{C}^{\text {sens } 2}=40 \mathrm{~ms}$

The results show that the influence of $t_{P}^{t t a 1}$ and $t_{P}^{t t a 2}$ is sufficiently small and can be neglected.

\section{CONCLUSION}

We have performed the optimization of a sensor fusion system consisting of two sensors, a bus system and a fusion/prediction core with respect to the schedule parameter space $\left(t_{C}^{\text {sens } 1}, t_{C}^{\text {sens } 2}, t_{P}^{\text {sens }}, t_{C}^{t t a}, t_{P}^{t t a 1}, t_{P}^{t t a 2}, t_{C}^{\text {pre }}, t_{T}^{\text {pre }}\right.$ and $\left.t_{P}^{\text {pre }}\right)$. The area of the covariance ellipse was chosen as the optimization criterion. We have shown that by a judicious choice of selected schedule parameters that are adjustable at no or little cost the fusion accuracy can be improved. The time interval between the state time and the real time at which an application expects a new image of the environment was identified as the dominant factor on fusion accuracy. This means that for analytically tractable OOSM algorithms such as the buffering approach the optimization can be performed on the basis of explicit formulae (see also [19]) without model dependent simulations.

\section{APPENDIX}

The example that is used in this paper corresponds to the one used in [10]. It is governed by the state transition matrix

$$
F\left(t_{k+1}-t_{k}\right)=\left[\begin{array}{cc}
1 & \left(t_{k+1}-t_{k}\right) \\
0 & 1
\end{array}\right]
$$

where the state consists of position and velocity of one coordinate. The dynamical system is corrupted by a zeromean, white, discretized continuous time process noise with covariance matrix $Q_{t_{k}}$

$$
Q_{t_{k}}=\left[\begin{array}{ll}
\frac{\left(t_{k+1}-t_{k}\right)^{3}}{3} & \frac{\left(t_{k+1}-t_{k}\right)^{2}}{2} \\
\frac{\left(t_{k+1}-t_{k}\right)^{2}}{2} & t_{k+1}-t_{k}
\end{array}\right] \cdot q
$$

with $q=0.5 \frac{m^{2}}{s^{3}}$ being the power spectral density of the continuous time process noise. The measurement matrix for both sensors is

$$
H_{t_{k}}^{\text {sensor } 1 / 2}=\left[\begin{array}{ll}
1 & 0 \\
0 & 1
\end{array}\right]
$$

i. e. both position and velocity are assumed to be measurable. The measurement noise of both sensors is characterized by the diagonal covariance matrix

$$
R_{t_{k}}^{\text {sensor } 1 / 2}=\left[\begin{array}{cc}
1 m^{2} & 0 \\
0 & 0.1 \frac{m^{2}}{s^{2}}
\end{array}\right] .
$$

\section{REFERENCES}

[1] A. Gelb, J. Kasper, R. Nash, C. Price, and A. Sutherland. Applied Optimal Estimation. The M.I.T. Press, 1974.

[2] S. Thomopoulos and L. Zhang. Distributed filtering with random sampling and delay. Proceedings on the 27th Conference on Decision and Control, December 1988.

[3] Y. Bar-Shalom. Multitarget-Multisensor Tracking: Applications and Advances, volume 3 of Multitarget-Multisensor Tracking. Artech House, 2000.

[4] FlexRay Consortium. Flexray communications system. Protocol specification, December 2005. Version 2.1 Revision A.

[5] F. Hartwich, B. Müller, T. Führer, and R. Hugel. Time triggered communication on CAN. In Proceedings 7th International CAN Conference, Amsterdam, The Nederlands, 2000. 
[6] TTAGroup. Specification of the TTP/C Protocol. TTAGroup, 2003. Available at www.ttagroup.org.

[7] H. Kopetz et al. Specification of the TTP/A protocol. Research Report 61/2002, Vienna, Austria, September 2002. Version 2.00.

[8] N. Kaempchen and K. Dietmayer. Data synchronization strategies for multi-sensor fusion. In Proceedings of the IEEE Conference on Intelligent Transportation Systems, October 2003.

[9] T. D. Larsen, N. K. Poulsen, N. A. Andersen, and O. Ravn. Incorporation of time delayed measurements in a discrete-time kalman filter. Proceedings of the 37th Conference on Decision and Control, Tampa, Florida, USA, pages 3972-3977, 1998.

[10] Y. Bar-Shalom, H. Chen, and M. Mallick. One-step solution for the multistep out-of-sequence-measurement problem in tracking. IEEE Transactions on Aerospace and Electronic Systems, 40(1), January 2004.

[11] Y. Bar-Shalom and H. Chen. IMM estimator with out-of-sequence measurements. IEEE Transactions on Aerospace and Electronic Systems, 41(1), January 2005.

[12] M. Mallick and A. Marrs. Comparison of the KF and particle filter based out-of-sequence measurement filtering algorithms. 6th International Conference on Information Fusion, pages 422-430, 2003.

[13] M. Orton and A. Marrs. Particle filters for tracking with outof-sequence measurements. IEEE Transactions on Aerospace and Electronic Systems, 41(2), April 2005.

[14] K.-S. Zhang, X. R. Li, and Y.-M. Zhu. Optimal update with outof-sequence measurements. IEEE Transactions on Signal Processing, 53(6), JUNE 2005.

[15] S. Maskel, R. Everitt, R. Wright, and M. Briers. Multi target out-ofsequence data association. Conference of Information Fusion, 2004.

[16] S. Challa, R. Evans, and X. Wang. A bayesian solution and its approximations to out-of-sequence measurement problems. Information Fusion(4):pages 185-199, 2003.

[17] X. Wang and S. Challa. Augmented state IMM-PDA for OOSM solution to maneuvering target tracking in clutter. Proceedings of Radar 2003 International Conference, pages 479-485, September 2003. Adelaide, Australia.

[18] Y. Anxi, L. Diannong, H. Weidong, and D. Zhen. A unified out-ofsequence measurements filter. IEEE International Radar Conference, pages $453-458$, May 2005 .

[19] M. Mauthner, W. Elmenreich, A. Kirchner, and D. Boesel. Outof-sequence measurements treatment in sensor fusion applications: Buffering versus advanced algorithms. Proceedings of the 4. Workshop Fahrerassistenzsysteme Hoesslinsuelz/Loewenstein, October 2006. 